

Review Paper

\title{
Industrial applications of green chemistry: Status, Challenges and Prospects
}

\author{
Rajni Ratti ${ }^{1}$
}

Received: 13 July 2019 / Accepted: 9 January 2020 / Published online: 23 January 2020

(c) Springer Nature Switzerland AG 2020

\begin{abstract}
Green Chemistry is expanding its wings from academic laboratories to industrial units. Sustainable practices include replacement of volatile organic solvents which constitute the bulk of a reaction material, developing recyclable catalysts, developing energy efficient synthesis and encouraging the use of renewable starting material. By following the principles of green chemistry, turn-over of many companies have increased immensely leading to both environmental as well as economic benefits. This review explores various examples wherein green chemistry has enhanced the sustainability factor of industrial processes immensely and suggests the measures which should be taken to promote as well as popularize the green practices in synthesis.
\end{abstract}

Keywords Sustainable synthesis · Recyclable catalysts · Turn-over number · Solvents · Bio-catalysis

\section{Introduction}

The 20th century has seen a phenomenal growth of global economy and a continuous improvement of standard of living in the industrialized countries. The increasingly competitive economic outlook and the shrinking graph of natural resources on the planet pose an urgent need to reduce the energy expenditure as well the production of waste. Sustainability is one of the main drivers for innovations in order to allow the technical industries to work for the well-being of consumers in a safe and healthy environment. The most attractive concept towards achieving sustainability is "Green Chemistry" - a term coined at United States Environmental Protection Agency by Anastas and Warner [1], and is defined as the utilization of a set of principles that reduces or eliminates the use or generation of hazardous substances in the design, manufacture and application of chemical products [2-4]. The term 'hazardous' is used in its broadest context which includes physical (e.g. explosive, flammable), toxicological (e.g. carcinogenic, mutagenic) and global (e.g. ozone depletion, climate change) factors. The tools of green chemistry are alternative feedstock, solvents and reagents, and catalytic versus stoichiometric processes. Developing green methodologies is a challenge that may be viewed through the framework of the "Twelve Principles of Green Chemistry" (Designed by P.T. Anastas and Warner).

\section{The twelve guiding principles of green chemistry}

Since its inception in early 1990s, green chemistry has grown into a significant, internationally engaged focus area within chemistry [5-8]. Green chemistry is basically a proactive approach aimed at designing a synthesis/process in a sustainable way right from the beginning. Preventing waste formation rather than devising methods to cleaning it up, developing atom efficient technologies based on renewable feedstock using minimum energy requirements and inherently safer chemicals, discouraging the use of volatile organic solvents and replacing them by greener alternatives are the main aims of green chemistry. To develop sustainable

Rajni Ratti, rajniratti@gmail.com | 'SGGS Khalsa Colllege, Mahilpur, Punjab, India. 
processes stoichiometric reagents should be replaced by catalytic reagents, end products should be bio-degradable and analytical methodologies should allow real time in-process monitoring. It is not always possible to incorporate all the principles in a particular process, however efforts should be made to follow as many principles as possible.

\section{Industrial applications of green chemistry}

Green Chemistry is not a lab-curiosity; instead it aims at big objective of creating a sustainable tomorrow. Increasing number of green methodologies developed by academic and industrial researchers enables companies to commercialize these ideas. Industry, from small businesses to large corporations, has already made strategic moves towards sustainability by adopting the principles of green chemistry. The development of less hazardous processes and commercial products, the shift from inefficient chemical routes towards bio-based synthesis, and the replacement of oil-based feed stocks by renewable starting materials are only a few examples of the major decisions taken that will ultimately have vast consequences for the world chemical markets.

As per the analysis of Environmental Protection Agency, the US drug industry has decreased the use of VOCs by $50 \%$ between 2004 and 2013 by adopting principles of green chemistry. In the same time span, the amount of chemical waste released to air, land and water decreased by $7 \%$ as per Toxics Release Inventory (TRI) of EPA.



Reprinted in part from C\&EN, 201593 (5) 32-33
Recently four industrial drug units located in Hyderabad region of India has been closed on account of creating pollution [9]. China, too, has strict environmental concerns and has taken regulatory action on $40 \%$ of the industrial units located in thirty provinces [10]. These changes in policy suggest that it has become imperative to follow green practices.

Plastics, in spite of several uses, have a bad reputation owing to their origin from polymers derived from non-renewable petrochemicals and their non-biodegradable nature. However, the same can be made from renewable feedstock as shown by a study carried out by Utrecht University [11]. Studies by Utrecht University also show that the market of bio-plastics will grow by approximately 37\% per year till 2013 and at a rate of $6 \%$ between 2013 and 2020. Many marketing hubs have joined the initiative to replace plastics with bio-plastics. Wal-Mart has been using bio-plastics in packaging wherever possible [12]. On similar lines Nokia, a mobile making company, used 50\% bio-plastics in Nokia 3111 Evolve phone cover as well as in Nokia C7 phone [13].

Procter \& Gamble replaced most of the PVC based materials with greener alternatives [14]. Along with other companies $P \& G$ have taken the initiative to develop new solvents so as to replace volatile organic carbons in glossy paints.

Greener synthesis of Ibuprofen launched by BASF involves half the number of steps as compared to traditional method. Atom efficiency of new process is almost double than the old synthesis. In pursuit for the development of sustainable methodologies, BASF developed BASIL $^{\text {TM }}$ (Bi-phasic Acid scavenging utilizing ionic liquids) process involving the production of generic photo initiator precursor alkoxyphenylphosphine [15]. Using this technology the yield increased from 50 to $98 \%$.

The Warner Babcock institute for Green Chemistry has developed a green hair-dye "Hairprint" which is a nontoxic, vegetable based product providing an alternative to the toxic, skin irritating and carcinogenic dyes [16].

USA based Merck \& Co., Inc. has successfully applied the principles of green chemistry to the synthesis of antiviral drug (cytomegalovirus infection) Letermovir which is currently in phase III of clinical trials. Cytomegalovirus (CMV) is a common virus whose infections are generally asymptomatic in healthy individuals but can cause severe damage in patients with immuno-depressed systems. The importance of this drug can be judged from the fact that it has been granted Fast-Track status by FDA and Orphan product designation by European Medicine agency for the prevention of CMV viremia in high risk population.

An evaluation of its traditional synthesis scheme revealed several areas for improvements like a very low overall yield of $10 \%$ due to a late stage resolution to access 




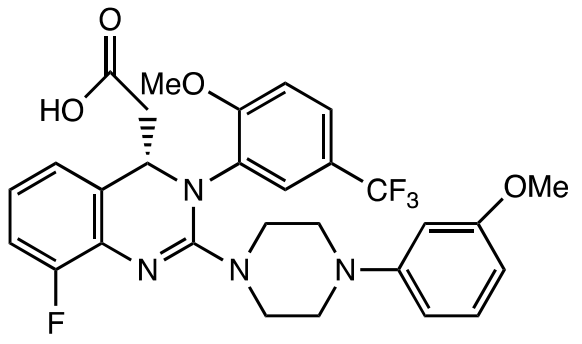

Letermovir

Scheme 1 Green synthesis of Letermovir

a stereogenic center, the use of nine different solvents, high palladium loading in Heck coupling. Moreover, no recycling of solvents and reagents had been there in the scheme.

Greener synthesis, as published by Merck, involves a novel cinchonidine based PTC-catalyzed Aza-Michael reaction for configuring the single stereocenter as shown in Scheme 1 [17]. Also, there is an increase in overall yield by $60 \%$, reduction in raw material cost by $93 \%$ and reduction in water usage by $90 \%$. It has been estimated that, once operational, this optimized process will lead to reduction of more than 15,000 MT of waste over the life time of Letermovir. Life-Cycle Assessment reveals that the green process is expected to decrease the carbon foot-print by $89 \%$. It is quite evident from the green synthesis of Letermovir that the Green Chemistry is not only environmentally friendly but also economically lucrative. This scheme has won the EPA's Presidential green chemistry award under the category "Greener synthetic pathways" in 2017 [18].

Of the various technologies used in green chemistry, biocatalysis holds an important place [19]. Most of the reactions occurring in physiological systems are catalyzed by enzymes which are nature's catalysts. Enzymes are not only biodegradable but are renewable as well due to the ease of production by fermentation of sugar etc. In order to achieve the aims of sustainability, more and more companies are working in the area of designing and using enzymes as biocatalysts. An impressive case highlighting the impact of biocatalysis on pharmaceutical manufacturing is the greener synthesis of Pregabalin, an active ingredient of neuropathic pain reliever Lyrica . In 2008, Pfizer improved the classical route for the synthesis of Pregabalin by adopting biocatalysis as a key step which led to $90 \%$ reduction in solvent usage, $50 \%$ reduction in the requirement of raw materials besides energy savings [20]. Solvent and energy saving in the process is equivalent to reducing 3 million tons of $\mathrm{CO}_{2}$ emissions which is actually equivalent to taking 1 million Indian cars off the road for a year. Schemes 2 and 3 compare the classical and greener route for the synthesis of pregabalin.

Not only in drug synthesis, biocatalysts also find important applications in the synthesis of plastics. Now a days, research is mainly targeted towards the synthesis of biodegradable plastics from renewable resources.

California based start-up "Newlight Technologies", founded in 2003, took a funding of $\$ 9.2$ million for developing a carbon negative technology that combines air with methane emissions to produce Aircarbon ${ }^{\mathrm{TM}}$ a

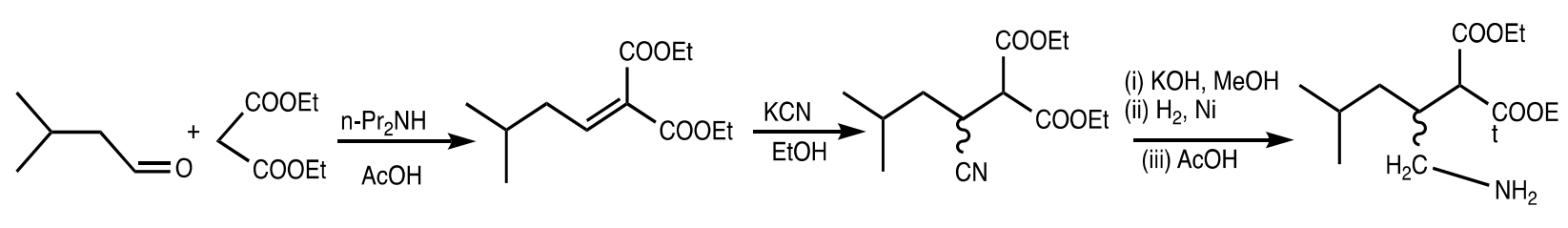

(S)-(+)- Mandelic acid

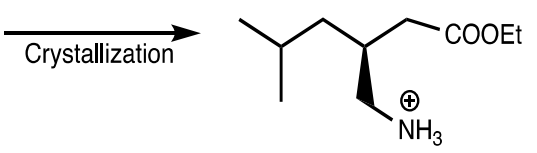<smiles>O=[N+]([O-])[C@H](O)c1ccccc1</smiles><smiles>CC(C)C[C@@H](CN)CC(=O)O</smiles>

Scheme 2 Conventional synthesis of Pregabalin 


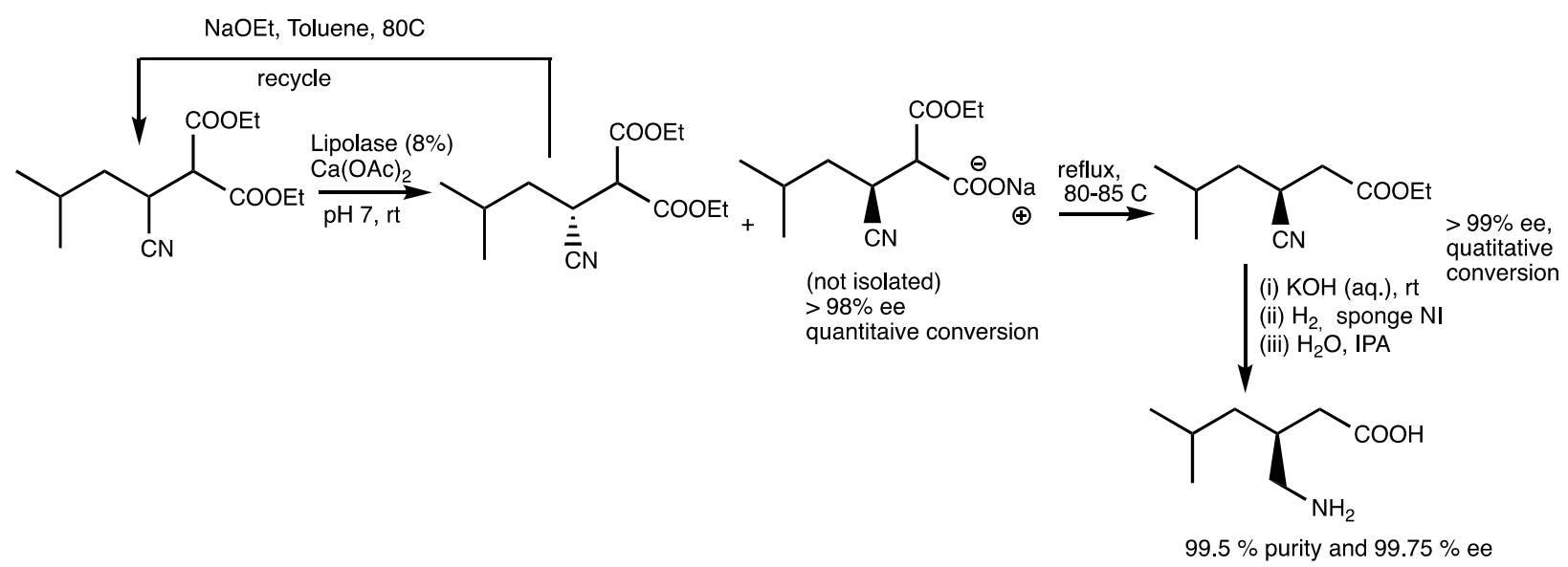

Scheme 3 Enzyme catalyzed synthesis of Pregabalin

thermoplastic. Aircarbon ${ }^{\mathrm{TM}}$ is approximately $40 \%$ oxygen from air and $60 \%$ carbon and hydrogen from methane emissions. The technology itself was not new but the use of a proprietary biocatalyst by Newlight Technologies made it actually commercially viable by increasing the yield nine times and decreasing the cost by a factor of three thereby making Aircarbon cheaper than oil based plastics. With the commercial scale-up in 2013, Aircarbon $^{\mathrm{TM}}$ was adopted by a number of leading brands like Dell, Hewlett-Packard, IKEA, Sprint, The Body Shop and Vinmar for manufacturing their respective products. In recognition of the company's commercial achievements, Newlight was named "Most innovative company of the year" in 2013 and Aircarbon ${ }^{\text {TM }}$ was named "Tech innovation of the year" by The American Business Awards [21]. For the green attributes of the process involving capturing and using greenhouse gases, Newlight technologies has been awarded the prestigious EPA's Presidential Green Chemistry Challenge award in 2016 [22].

Most chemical processes involve solvents in the reaction and separation step to dissolve solids, reduce viscosity, modulate temperature, and recover products by means of extraction or recrystallization as reaction media or for cleaning purposes. Solvents not only dissolve the reactants but they also affect the rates, chemo-, regioand stereoselectivities of reaction. However, majority of the organic solvents used in industry, despite their inherent advantages, are associated with several ill-effects on human health and environment. Moreover, these solvents are derived from non-renewable resources like petroleum. These parameters are in contradiction to the very basics of Green Chemistry. Due to these reasons, the only alternative available is to substitute these environmentally harmful solvents with some benign solvents. Hungerbuhler et al. [23] discussed the following four directions towards the development of green solvents
1. Substitution of hazardous solvents with one that show better EHS (Environment, Health, Safety) properties such as increased biodegradability or reduced ozone depletion potential [24].

2. Use of "bio-solvents" i.e. solvents produced from renewable resources such as ethanol produced by fermentation of sugar-containing feeds, starchy feed materials or lignocellulosic materials [25].

3. Substitution of organic solvents with supercritical $\mathrm{CO}_{2}$ in polymer processing avoids the use of chlorofluorocarbons, and reduces the ozone depletion [26].

4. With ionic liquids that show low or negligible vapour pressure, and thus fewer emissions to air [27].

Fabric dyeing consumes a lot of water. About 7 gallons of water is used up to dye a T-shirt and lot of energy is wasted in drying the dyed material. A Dutch start-up recently launched water-free dyeing using supercritical carbon dioxide as a solvent under pressure and at elevated temperature. As no water is used so energy required in drying is also saved [28].

Elevance Renewable Sciences, Inc., used a nobel prize winning metathesis technology developed by Grubb's to produce two green solvents

In collaboration with the surfactant manufacturer Stepan, Elevance produced a surfactant called STEPOSOL MET-10U as a replacement for $\mathrm{N}$-methyl pyrrolidone and dichloromethane in adhesive removers and paint strippers. This surfactant can also be used in household and industrial cleaners in place of glycol ethers. STEPOSOL MET-10U is a unique unsaturated di-substituted amide derived from a bio-based feedstock [29]. With a KauriButanol value greater than 1000, STEPOSOL MET-10U provides superior cleaning performance and is environmentally friendly due to a low vapor pressure, high boiling point, and Biorenewable Carbon Index (BCl) of 75\%. 
Another heavy-duty green degreasing solvent developed by Elevance Renewable Sciences is Elevance Clean ${ }^{\mathrm{TM}}$ 1200 which is a VOC free bio-based solvent [30]. In 2015, Elevance $\mathrm{Clean}^{\mathrm{TM}} 1200$ was awarded bio-based product innovation of the year at WBM bio business awards for its out-standing cleaning performance. Being produced from natural oils this non-flammable solvent meets the various restrictive environmental regulations. Therefore, Elevance Clean $^{\text {TM }} 1200$ is

(i) VOC exempt (Directive 2004/42/CE of the European Parliament and the Council)

(ii) $\mathrm{REACH}$ registered

(iii) Readily biodegradable (by OCED method)

(iv) Free of components listed in the EU dangerous substances directive (Regulation No. 1272/2008).

The various advantages of Elevance Clean ${ }^{\mathrm{TM}} 1200$ are enlisted below

(i) Strong solvency characteristics greater than even of d-limonene, dibasic esters, vegetable esters and isoparaffins on the Kauri butanol $(\mathrm{Kb})$ scale.

(ii) Excellent performance across a broad range of cleaning applications which includes metal cleaning, industrial and institutional degreasing, transportation and food processing.

(iii) Being non-flammable, it is easy to handle. It works very well in the neutral $\mathrm{pH}$ range (6-9) thereby eliminating the need of caustic cleaning products.

In 2014, Solberg Company won the first Insight Innovation award at the $3^{\text {rd }}$ annual THINC for its environmentallyfriendly fire-fighting foam concentrate RE-HEALING. Conventional firefighting foams use fluorinated surfactants which are hazardous for the environment. The RE-HEALING firefighting foam concentrate use a blend of non-fluorinated surfactants, sugars, solvent and corrosion inhibitor leading to far less environmental impacts. Control, extinguishing time, and re-ignition resistance are necessary for the safety of fire-fighters and RE-HEALING fulfills all these conditions. The company also won the 2014 EPA Presidential Green Chemistry award for this innovation. [31].

Using catalytic reagents over stoichiometric reagents is one of the principles of green chemistry. Developing recyclable and recoverable catalyst adds to the green profile of a technology. Exhausts from the automobile engines pose a major threat to the environment. Inside the engine, temperature being very high, oxygen and nitrogen react to form nitric oxide (NO). Conversion of $\mathrm{NO}$ to $\mathrm{NO}_{2}$ is highly desirable for the removal of oxides of nitrogen. However, this reaction is, in general, quite slow. A team of scientists from U.S, China and South Korea developed the catalyst using $\mathrm{Mn}$-Mullite (Sm, Gd) $\mathrm{Mn}_{2} \mathrm{O}_{5}$-manganese-mullite materials containing either Samarium or Gadolinium to convert the toxic diesel engine exhaust product nitric oxide to a more benign nitrous oxide [32]. Over a range of temperatures, the new catalyst performed better than platinum (around $64 \%$ better at $300{ }^{\circ} \mathrm{C}$ and $45 \%$ better at $\left.120^{\circ} \mathrm{C}\right)$.

RCHEM Pvt. Ltd. Hyderabad, in collaboration with Chaudhuri et al. [33] developed a green synthesis of antiulcer drug Ranitidine. The conventional synthesis generates dimethylsulfide which is a hazardous to human health. Prof. Chaudhuri, from IIT Guwahati, and Prof. Kantam from IICT Hyderabad developed vanadium-titanium and titanium phosphorous based solid supported catalysts. In the presence of these heterogeneous catalysts $\mathrm{H}_{2} \mathrm{O}_{2}$ acts as an oxidant to convert the dimethylsulfide to colourless odourless liquid dimethyl sulfoxide (DMSO). The DMSO generated is further used in the manufacturing process of drug thereby reducing the cost of production by $20 \%$.

There are numerous applications where green chemistry has marched beyond the research laboratories and finding commercial applications. However, a lot more efforts are required, particularly in the area of life-cycle analysis so as to evaluate the environmental impact of the various "green" drugs after these traverses the human physiological system. Terry Collins, from the University of Pittsburg, developed a series of tetra-amido macrocyclic ligand based catalysts modelled on peroxidase enzymes [34]. Collins proposed that addition of these at a late stage in the sewage treatment process could help break down a wide variety of chemical residues from the drugs before they can affect the environment.

\section{Challenges}

Just being green is not enough for a process to be a commercial success. Regulatory, economic, political and technical challenges often impede the industrial implementation of a green process.

Current regulations are focused on reducing risk through reductions in exposure while green chemistry promotes the reduction of inherent risk by reduction of hazard. In U.S, the regulations require that every time a manufacturer changes the production process, it has to undergo a re-certification process with the FDA. This process is both costly and time- consuming, and hence serves to dissuade firms that would otherwise invest in developing atom efficient chemistries that reduce waste. Changes to more benign processes are inhibited by costintensive, control-oriented regulation. Lack of awareness among the different stake-holder groups poses a barrier to 
the implementation of green processes. Developing a successful green process is not only about green chemistry, it involves the knowledge of green engineering, biotechnology, economics and above all toxicology. The chemists generally lack the training in these disciplines which further hampers the implementation of green chemistry on an industrial scale. Even if all factors are the in the favour of a green process, it can be rejected on a commercial-scale if it fails to be economically attractive. Green industrial processes should be comparable to the traditional processes in terms of costs of the products.

There are a number of examples of technically robust, environmentally-friendly processes that have been started at first but were withdrawn at a later stage due to commercial implications. It does not always pay to be green in the chemicals sector. Thomas Swan and Company in Consett, UK, implemented the work of Martyn Poliakoff (Nottingham University), to start world's first continuous-flow reactor using supercritical carbon dioxide as a solvent [35]. Sc- $\mathrm{CO}_{2}$ system lead to selective hydrogenation of isophorone to 3,3,5-trimethylcyclohexanone without any by-product formation. This lead to elimination of an expensive and energy-intensive separation required by the conventional technique. But due to the lack of government subsidies, the plant could not provide chemicals more cheaply than those made by the traditional non-green methods. Therefore, after commercially running from 2002 to 2009 , this plant was taken out of production.

Similar things happen with the process involving isomerization of 3,4-epoxybut-1-ene to 2,5-dihydrofuran in a phosphonium iodide ionic liquid developed by Eastman Chemical Company.

Capital investment also prevents the commercialization of a green technology. IFP (France) used ionic liquids, as solvent as well as co-catalyst, on a large scale for the nickel catalyzed dimerization of alkenes, named as Difasol process [36]. This is a biphasic process wherein the product forms a separate layer above the ionic liquid layer and thus can be easily separated. Compared to the conventional Dimersol process, this method has many advantages like better catalytic activity, ease of separation of product, better dimer selectivity and higher reactor space time yields. However, the cost of capital equipment posed a hurdle towards its commercial implementation.

The commercialization of green processes also requires many changes in all part of the long and global supply chain. Eden Organic foods developed a BPA-free coating for food packaging which was found to be compatible with some foods like beans but not for highly acidic tomato sauce. Switching to different coating type for different food type implies a smaller market size and change in manufacturing machines and consequently a higher cost. The implementation of such initiatives requires that everyone in the value chain agrees and is willing to accept the changes.

As most of the industries have been driven by monetary profits therefore voluntary adoption of the sustainable practices seems less feasible. A strong, attractive and balanced regulation is required so as to enforce the greener practices. The most promising and significant regulation is the REACH (Registration, Evaluation, Authorization and Restriction of Chemical substances) regulation framed and launched by European Union in 2007 [37]. On one hand REACH makes it mandatory for the chemical companies to disclose more information on the environmental and health risks of their products; on the other hand it grants potential exemptions on registration for five years for a process which favours new sustainable innovation. This move of European Union has motivated other countries to devise similar regulations so as to create a sustainable chemical industry.

\section{Prospects}

Green chemistry holds the key to a sustainable society. It has the inherent potential to bridge the gap between society and science. Innovations, backed by sound policies and regulations, will accelerate the large-scale implementation of green processes. Next generation of chemists should be taught the basics of green chemistry at a very early stage so that they can think green and develop safer methodologies. Interdisciplinary and multidisciplinary research can help in solving the various technical hurdles for commercializing this philosophy. Subsidizing the greener initiatives and tax exemptions to the companies adopting green processes will have a positive impact. Industries should realize the fact that getting a new greener process registered and making capital investment is a one-time investment which can have positive impacts on various aspects of society and environment. Collective and sincere efforts by researchers, engineers, corporates and policymakers can actually make the chemistry Green.

Funding No funding has been received by the author for this work.

\section{Compliance with ethical standards}

Conflict of interest The author declares that there is no conflict of interest.

\section{References}

1. Anastas PT, Warner JC (1998) Green chemistry: theory and practice. Oxford University Press, Oxford 
2. Anastas PT, Williamson TC (1998) Green chemistry: frontiers in benign chemical syntheses and processes. Oxford University Press, Oxford

3. Kidwai MM (2006) Pure Appl Chem 78:1983-1992

4. Kirchhoff M (2005) Resour Conserv Recycl 44:237-243

5. Clark JH (2006) Green Chem 8:17-21

6. Manley JB, Anastas PT, Cue BW Jr. (2008) J Clean Prod 16:743-750

7. Keith LH, Gron LU, Young JL (2007) Chem Rev 107:2695-2708

8. Mason BP, Price KE, Steinbacher JL, Bogdan AR, McQuade DT (2007) Chem Rev 107:2300-2318

9. Reddy U (2018) PCB cracks down, shuts four polluting industries. The Times of India. http://www.pharmabiz.com/NewsD etails.aspx?aid $=116312 \&$ sid $=1$

10. Mullin R (2018) Drug chemical makers brace as china cracks down on pollution. Chem Eng News 96:23-25

11. Shen L, Haufe J, Patel MK (2009) Product overview and market projection of emerging bio-based plastics. PRO-BIP 2009. In: Report commissioned by European Polysaccharide Network of Excellence and European Bioplastics. Copernicus Institute for Sustainable Development and Innovation, Utrecht University, Netherlands

12. https://www.walmartsustainabilityhub.com

13. https://www.go-green.ae/greenstory_view.php?storyid $=1280$

14. https://en-ae.pg.com/sustainability-reports/ Procter and Gamble sustainability report 2010

15. BASF SE (2003) Methods for the separation of acids from chemical reaction mixtures by means of ionic fluids. World Patent WO/2003/062251

16. https://www.warnerbabcock.com/scientific-discovery-reversesgray-hair-natural-color-invented-warner-babcock-institute/

17. Humphrey GR, Dalby SM, Andreani T, Xiang B, Luzung MR, Song ZJ, Shelvin M, Christensen M, Belyk KM, Tschaen DM (2016) Org Process Res Dev 20:1097-1103

18. www.epa.gov/greenchemistry/green-chemistry-challenge2017-greener-synthetic-pathways-award

19. Tao J, Xu J-H (2009) Curr Opin Chem Biol 13:43-50

20. Martinez CA, Hu S, Dumond Y, Tao J, Kelleher P, Tully L (2008) Org Process Res Dev 12:392-398

21. http://stevieawards.com/aba/newlight-technologies-compa ny-year
22. https://www.epa.gov/greenchemistry/presidential-green-chemi stry-challenge-2016-designing-greener-chemicals-and-specific

23. Capello C, Fischer U, Hungerbuhler K (2007) Green Chem 9:927-934

24. Gani R, Gonzalez CJ, Kate A, Crafts PA, Jones M, Powell L, Atherton JH, Cordiner JL (2006) Chem Eng 1:30-41

25. Savaiko B (2004) A promising future for ethanol. World Ethanol Biofuels Rep 2:20-22

26. Noyori R (1999) Chem Rev 99:353-354

27. Petkovic M, Seddon KR, Rebelo LPN, Pereira CS (2011) Chem Soc Rev 40:1383-1403

28. http://www.dyecoo.com/pdfs/colourist.pdf

29. http://www.stepan.com/uploadedFiles/Literature_and_Downl oads/General_Lit/Household,_Institutional,_and_Industrial Cleaning/STEPOSOLMET10UGreenChemistryGuide.pdf

30. G Hategan, C Shaner, R Littich, S Block, M Morie-Bebel (2015) $\mathrm{H} \& \mathrm{PC}_{\text {today }} 10$. https://elevance.com/product/elevance-clean $-1200 /$ ?prod-id=109\&cat-id=cleaning-17

31. https://www.epa.gov/greenchemistry/presidential-green-chemi stry-challenge-2014-designing-greener-chemicals-award

32. Wang W, McCool G, Kapur N, Yuan G, Shan B, Nguyen M, Graham UM, Davis BH, Jacobs G, Cho K, Hao X (2012) Science 337:832-835

33. Bora U, Chaudhuri MK, Dehury SK (2002) Curr Sci 82:1427-1436

34. Ellis WC, Tran CT, Denardo MA, Fischer A, Ryabov AD, Colllins TJ (2004) J Am Chem Soc 6:43-48

35. Licence P, Ke J, Sokolova M, Ross SK, Poliakoff M (2003) Green Chem 5:99-104

36. Favre $F$, Forestiere $A$, Hugues $F$, Olivier-Bourbigou $H$, Chodorge JA (2005) Oil Gas Eur Mag 31:83-87

37. https://ec.europa.eu/environment/chemicals/reach/reach _en.htm

Publisher's Note Springer Nature remains neutral with regard to jurisdictional claims in published maps and institutional affiliations. 